\title{
Correction to: Half a Century of Stereotyping Associations Between Gender and Intellectual Ability in Films
}

\author{
Ramiro H. Gálvez ${ }^{1,2}$ • Valeria Tiffenberg ${ }^{1}$ - Edgar Altszyler ${ }^{1,2,3}$
}

Published online: 11 April 2019

(C) Springer Science+Business Media, LLC, part of Springer Nature 2019

\section{Correction to: Sex Roles}

$$
\text { https://doi.org/10.1007/s11199-019-01019-x }
$$

The original version of the article unfortunately contained errors.

In Fig. 3, panel b, the gray shades were captured on top of the lines instead as a background.

The original article has been corrected and the updated figure is shown below.

The online version of the original article can be found at https://doi.org/ 10.1007/s11199-019-01019-x

Ramiro H. Gálvez rgalvez@dc.uba.ar

1 Departamento de Computación, FCEyN, Universidad de Buenos, Aires, Buenos Aires, Argentina

2 Instituto de Ciencias de la Computación, CONICET-UBA, Buenos Aires, Argentina

3 Fundación Sadosky, Buenos Aires, Argentina 
a

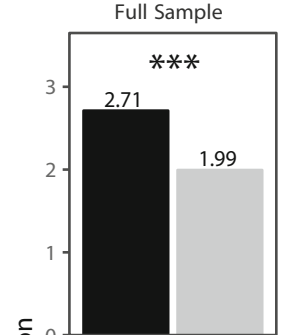

(

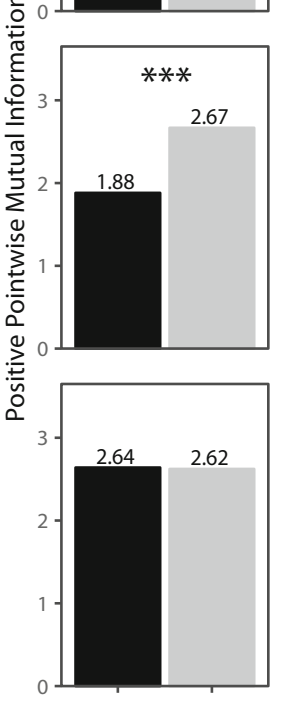

Female pronouns
Family/Animation
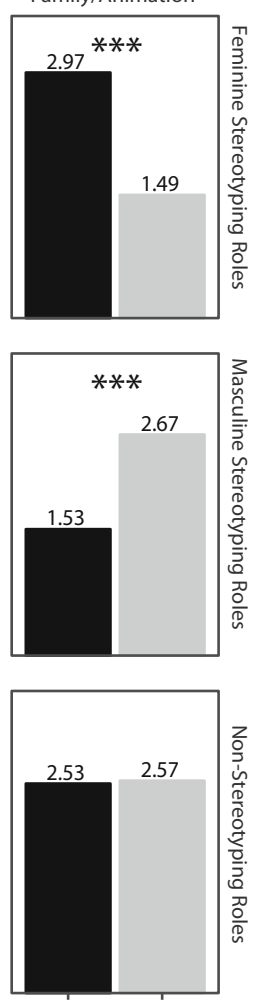

Male pronouns b
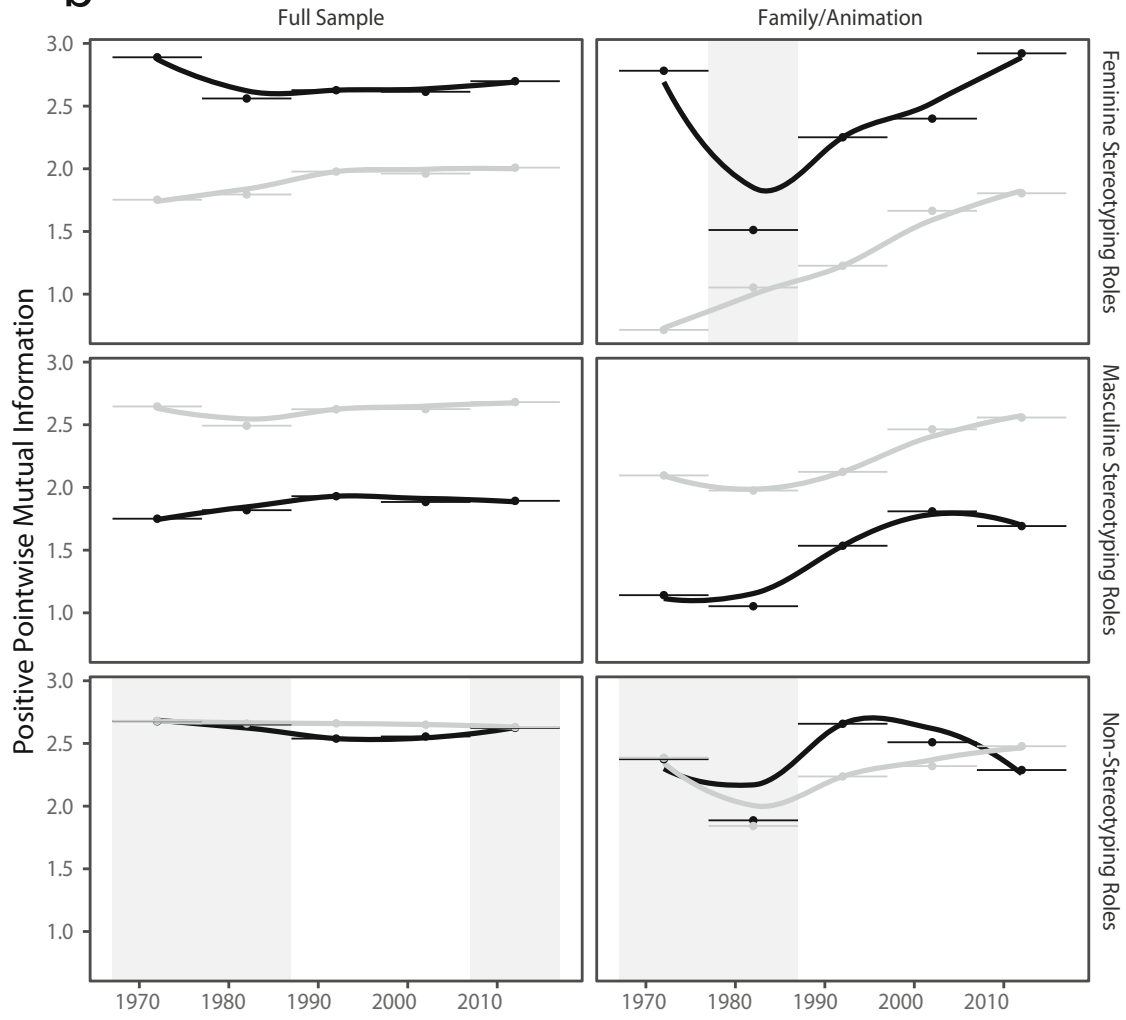

- Female pronouns

Male pronouns
Fig. 3 Word associations between gender pronouns and stereotyping roles. a Estimated associations between gender pronouns and stereotyping roles when analyzing films from 2010 up to and including 2016 for the full sample $(n=2902)$ and the family/animation subsample $(n=242)$. Asterisks indicate the results of log odds ratios statistical significance tests on the underlying contingency tables. b Time evolution of the estimated associations taking as input sets of films belonging to successive 10-year periods (1967-1976, 1977-1986, ..., 2007-2016). Tendencies are estimated through LOESS regressions. Gray areas indicate that, according to log odds ratios statistical significance tests, differences are not significant at the $5 \%$ level. $* * * p<.001$

Publisher's Note Springer Nature remains neutral with regard to jurisdictional claims in published maps and institutional affiliations. 